\title{
Deep Analysis of a Basic Traffic Model
}

\author{
Diego Uribe, Luis Lugo, Enrique Cuan \\ Instituto Tecnológico de la Laguna, \\ División de Estudios de Posgrado e Investigación, Torreón, Coahuila, \\ Mexico \\ \{diego, lclr, kcuan\}@itlalaguna.edu.mx
}

\begin{abstract}
In this article we analyze the basic elements to be considered in vehicular traffic by using modeling based on agents. One of the most important activities of a traffic engineer is to measure the capacity of a road in order to optimize the road infrastructure of a city. The incorporation of the microscopic and macroscopic characteristics of vehicular traffic flows are the key elements for developing a realistic model for measuring the capacity of a particular road. By using a basic traffic model, we present, on the one hand, the empirical relationships of a vehicular traffic system in terms of density, flow and speed; on the other hand, we show how the use of agent-based modeling to simulate vehicular traffic system proves to be a plausible alternative. Also, this work makes clear the shortcomings of a basic traffic model that must be considered for developing a more realistic traffic simulation system.
\end{abstract}

Keywords: Agent-based modeling, traffic model, micro and macro levels.

\section{Introduction}

Nowadays, heavy traffic in big cities is the cause of many urban problems: wasted time, poor fuel efficiency, high pollution, stress and unfortunately, an increase in the accident rate [2]. So the need for a network of intelligent transportation system to be able to cope with these multiple problems is evident.

Among the multiple tasks of traffic engineers, traffic flow analysis is perhaps the most important activity. Indeed, the optimization of the transport infrastructure largely depends on a through analysis of the traffic flow detecting areas and time periods of increased traffic density is critical for making decisions such as expanding the number of lanes, installing traffic lights, or altering the scheduled times of existing traffic lights.

Likewise, among the multiple tools available to the traffic engineer, a vehicular traffic simulator is an essential tool because it allows analyzing hypothetical scenarios corresponding to several possible vehicular flows. Also, this tool is essential as facilitates the estimation of real-time road traffic information. In fact, by using a vehicular traffic simulator, a traffic engineer embarks on preanalysis of the urban area to be investigated. The purpose of this activity is 
to establish various hypotheses to be verified, or disproved, based on the road traffic information obtained in real time.

According to the definition of a complex system as a system characterized by an environment in which multiple individual and independent elements interact with each other giving rise to an emerging phenomenon $[3,5]$, to analyze vehicular traffic from this perspective is rather a plausible approach. In fact, taking into account the multiple factors to be considered when driving a vehicle (e,g. the presence of other vehicles, road conditions, traffic lights, traffic accidents, pedestrian crossing), a traffic system may also be characterized as a complex system.

In this way, since agent based modeling is a computational methodology that allows us to model complex systems [15], we analyze in this work the basic elements to be considered in vehicular traffic by using modeling based on agents. To be more specific, the vehicles are represented as agents, whereas the road is represented as the environment in which the vehicles travel and interact. The micro and macro level that characterize a complex system are also analyzed by using a basic traffic model based on agents in order to present the empirical relationships of a traffic system in terms of density, flow and speed.

Also, as a fundamental part of our analysis work we point out the shortcomings of the traffic model that must be considered for developing a more realistic traffic simulation system. Since velocity is an intrinsic parameter to any traffic model, an appropriated representation of distance and time is essential for a traffic simulation system [8]. In other words, taking care of the distance is essential for a safe control of the speed. Thus, its fundamental to study the representation of distance and time in this model.

The theoretical framework of the methodology adopted in the traffic model is described in section 3. As it is briefly mentioned lines above, the model represents the simulation of a vehicular traffic system based on agents so the properties, behaviour and interactions between autos is detailed. Then, the empirical relationships of a traffic system in terms of density, flow and speed are presented in section 4 as well as the basic parameters for an elemental runtime analysis. Related work as other models for describing traffic flows is also mentioned in the next section.

\section{Related Works}

In this section, we briefly narrate other models for describing traffic flows. Traffic flow theory is a scientific field relatively young. The first traffic problems in the past century were solved by the implementation of rule of thumb methods. However, at the beginning of the 1950s, the turning point in the field of traffic engineering came when Glen Wardrop described traffic flows by using mathematical representations [12].

The first popular traffic flow model, commonly known as the LWR model, was based on fluid dynamics, a discipline for studying fluids (liquids and gases) in motion [6,11]. In fact, traffic flow model makes use of numerical analysis 
and algorithms to solve and analyze problems that involved fluid flows. The interactions of liquids and gases are used as an analogy to model the interactions among vehicles.

Another interesting work was the traffic model implemented by Prigogine and Herman who drew an analogy between gas-kinetic and the velocity distributions [10]. It is basically a stochastic model of traffic flow in which the microgoals of each driver are modified by the interaction with other drivers. Then, the central idea is to predict how much the microgoals of the driver have been modified by making use of a kinetic equation defined in terms of the desire of the driver and the interactions with other vehicles.

Statistical physics represents a more recent framework in traffic flow modelling. Particularly, the cellular automata model developed by Nagel and Schreckenberg is based on particles systems where the cells have varying states over time [9].

\section{Theoretical Framework}

The theoretical framework that supports the basic traffic model to be analyzed in this article is described in this section. First, we briefly define the microscopic and macroscopic properties of traffic flow to study vehicular transport systems. Then, we present the essential concepts of agent-based modeling, the methodology for designing and building the basic traffic model.

\subsection{Microscopic and Macroscopic Properties of a Traffic Flow}

Broadly speaking, the drivers and vehicles, that is, the actors of a vehicular transport system denote the microscopic characteristics, whereas the interactions between drivers denote the macroscopic characteristics of traffic flows [7].

The microscopic properties of a traffic flow are described, first, in terms of a single vehicle, and then, by considering two consecutive vehicles. The main characteristic to describe a vehicle in a traffic lane are: length, position, velocity, and acceleration.

Driving safely requires to be aware of how close another car is. Thus, by considering two consecutive vehicles in a traffic lane, we have two very important properties:

- space headway: it is defined as the space gap between two consecutive vehicles: a follower and its leader (i.e. predecessor), that is measured from the followers rear bump to its leaders rear bump. Such space gap is usually expressed in metres.

- time headway: it is defined as the time to travel the space headway at the current velocity. Such time gap is usually expressed in secs.

The macroscopic properties of a traffic flow are considered in terms of an aggregate level of multiple vehicles in a traffic stream. We begin with density: 
a measure to determine how crowded a section of a road is. In other words, the concentration or density of traffic, denoted by $k$, is estimated by the following expression:

$$
k=\frac{n_{x}}{x},
$$

where $n_{x}$ denotes the number of vehicles in the section of a road to be measured, $x$ denotes the length of the section of a road, and $k$ is expressed in terms of veh $/ \mathrm{m}$.

The second macroscopic property is concerned with time: a temporal measure to determine the number of vehicles passing at a particular point of the road during a particular period of time. In other words, the flow of traffic, denoted by $q$, is estimated by the following expression:

$$
q=\frac{n_{t}}{t}
$$

where $n_{t}$ denotes the number of vehicles in a particular period of time, $t$ denotes a particular period of time, and $q$ is expressed in terms of veh/s.

Average speed is the third macroscopic property and it is concerned with multiple rather than individual speeds. There are two different ways to estimate the average speed: the spatial measurement and the temporal measurement [1]. Assuming $\mathrm{n}$ vehicles in a traffic stream where the particular speed of each vehicle is registered in a specific point and a particular time, the temporal measurement of the average speed, denoted by $u_{t}$, is estimated by the following expression:

$$
u_{t}=\frac{\sum_{i=1}^{n}\left(u_{i}\right)}{n}
$$

where $n$ denotes the number of vehicles, and $u_{i}$ denotes the speed of an individual vehicle.

\subsection{Agent-based Modeling}

The approach for developing the basic traffic model is based on one of the primary methodologies to study complex problems: agent-based modeling. A complex system is defined as a system characterized by an environment in which multiple individual and independent elements interact with each other giving rise to an emerging phenomenon $[3,5]$. The complexity of a system is observed in natural and social phenomena that are non-deterministic and have distributed mechanisms and control. Thus, to simulate the interactions among agents to recreate the macroscopic properties that emerge is fundamental to make sense of the phenomena to investigate.

Agent based modeling is a computational methodology that allows us to model complex systems [15]. More precisely, ABM is a computational modelling paradigm that enable us to describe a complex system in terms of agents, environment, and interactions. While agents denote the basic ontological unit of the model, the environment represents the world in which the agent lives. In this work, we make use of Agent based modeling to represent vehicular traffic 
flows. To be more specific, the vehicles are represented as agents, whereas the road is represented as the environment in which the vehicles travel and interact. The micro and macro leves that characterise a complex system are also analysed to present the empirical relationships of a traffic system in terms of density, flow and speed.

\section{Basic Traffic Model and Discussion}

The empirical relationships of a traffic system in terms of density, flow and speed are presented in this section. First, we describe the basic traffic model used to illustrate the relationships between the macroscopic traffic flow characteristics, and then, the section concludes with a discussion about the shortcomings of the traffic model that are essential to be considered for developing a more realistic traffic simulation system.

\subsection{Empirical Relationships of a Traffic System}

The traffic model used to exemplify the empirical relationships of a traffic system in terms of density, flow and speed is the model developed by Wilensky [13]. This model represents a traffic lane in which the velocity of the cars is a function in terms of the available space between them. In other words, an individual driver might accelerate o decelerate according to the distance between his vehicle and the next: the space headway. The model also proves how traffic jams emerges spontaneously from slow-moving vehicles creating a ripple effect backward.

Moreover, the traffic model used to illustrate the relationships of a traffic system has been developed with NetLogo [14]. NetLogo is a programming language used to create models based on agents and has also proved to be a well suited tool for modeling complex systems evolving over time. One of the most appealing features of NetLogo is the capacity to investigate the connections between the micro-level behavior of individuals and macro-level patterns that emerge from their interactions [4].

The relation between speed and density is shown in Figure 1. It can be noticed how the speed falls as the number of cars increases. In other words, we observe a negative correlation between speed and density: as density increases, speed decreases and vice versa. We can also describe this relation in terms of the drivers comfort: as long as the density in a particular road is lower, the driver travels at his desired speed. On the contrary, the speed range available to a driver is limited to the extent that the density on the road increases.

Figure 2 shows the relation between flow and density. In this case, two stages can be noticed: increasing and decreasing of the flow as traffic density rises. In other words, we observe at the beginning a positive correlation reaching the maximum of flow and then, a negative correlation: as density increases, flow decreases.

In this way, Figure 2 shows two essential points to determine how much traffic might be allocated in a road space. First, when flow is at its highest, we 


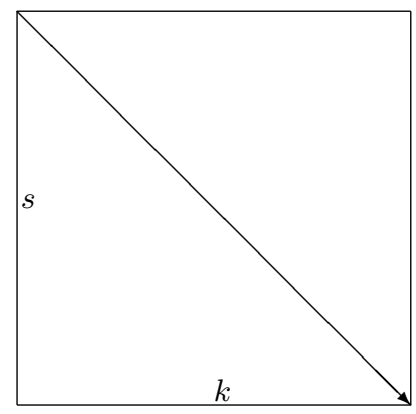

Fig. 1. The relation between speed $(s)$ and density $(k)$.

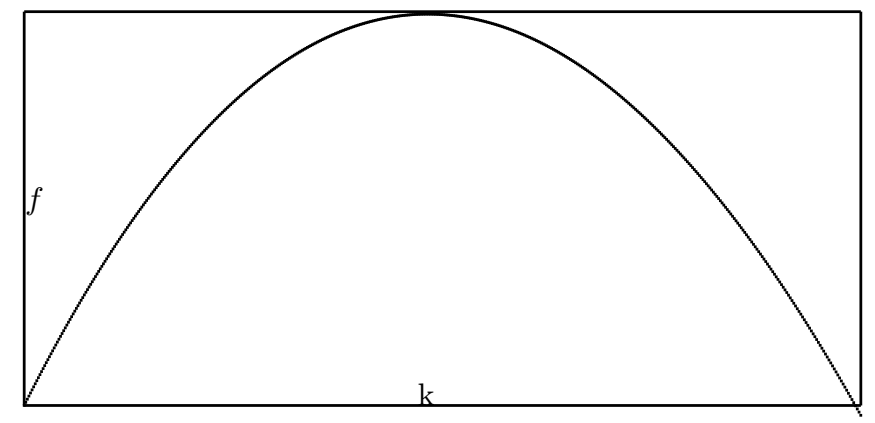

Fig. 2. The relation between flow $(f)$ and density $(k)$.

can consider this value to characterise the capacity of a particular road. Second, when density is at its highest value, we can consider this value to denote the emergence of a traffic jam.

Figure 3 shows the relationships between density, flow and speed by using the traffic model developed with NetLogo. Figures 3a, 3b, and 3c have been obtained with a density value of 10,20 , and 30 respectively. In other words, the traffic model has been run through different values of density. In this way, the relation between speed and density can be noticed: we see how speed is dropping whereas density increases.

From the perspective of the relationships between flow and density, the traffic engineer can define the capacity of the road in terms of a not very constrained speed. In other words, as long as the driver is able to accelerate to keep a target speed, previously defined by the traffic engineer, the flow is on the way to its highest point. Otherwise, the driver enters a state of deceleration that can eventually lead to a traffic jam. In this particular case, Figure 3 shows how a medium speed can be achieved with a density value of 20 . 


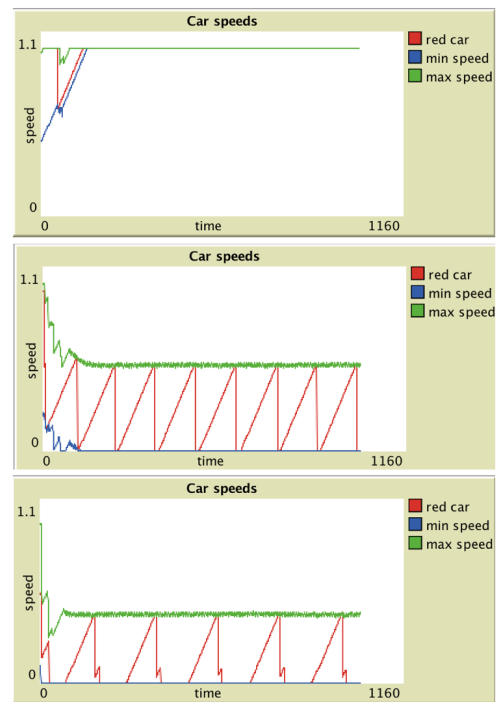

Fig. 3. The relationships between density, flow, and speed.

\subsection{Discussion}

Another fundamental part of our analysis work is the consideration of the shortcomings of the traffic model used to exemplify the relationships of a vehicular system. The purpose of further analysis of the model is to identify essential elements to be considered for developing a more realistic traffic simulation system.

Since velocity is an intrinsic parameter to any traffic model, an appropriated representation of distance and time is essential for a traffic simulation system [8]. As it was previously mentioned, the model depicts a traffic lane in which the velocity of the cars is determined according to the available space between them: a driver might accelerate o decelerate to the extent of the distance between his vehicle and the next. In other words, taking care of the distance is essential for the safety control of the speed. Thus, its fundamental to study the representation of distance and time in this model.

To achieve a better representation of the distance between cars we focus our attention in one of the fundamental microscopic traffic characteristics: the space headway. As we mentioned in section 3, the space headway between two consecutive vehicles: a follower and its leader (i.e. predecessor), is defined as the space gap measured from the followers rear bump to its leaders rear bump. Such space gap is usually expressed in meters [7].

In the case study discussed in this article, the traffic model developed with NetLogo measures the distance in terms of patches. A patch is the basic unit used by NetLogo to represent the environment in which the agents interact. In fact, NetLogo represents the environment (called "world") as a bi-dimensional space that is divided up into a grid of patches. 
Thus, being NeLogo a generic tool for agent-based modeling, the configuration of the environment, and therefore the definition and interpretation of a patche depends on the domain to be modelled. For example, in the classic Wolf-Sheep Predation model, the environment is represented by a landscape where a patche" denotes a minute portion of the landscape, that is, a minute portion of grass. On the other hand, in our case study model, the environment is represented by a road space where a "patche" denotes an undefined portion of the space headway, that is, an undefined measure of distance.

Even though the distance has not been properly defined, Figure 4 shows how the model allows us to observe the correlation between speed and space headway. When we run the model under the same density of traffic and different values of headway, speed gradually decreases as the headway increases, which denotes a more preventive driving habit.

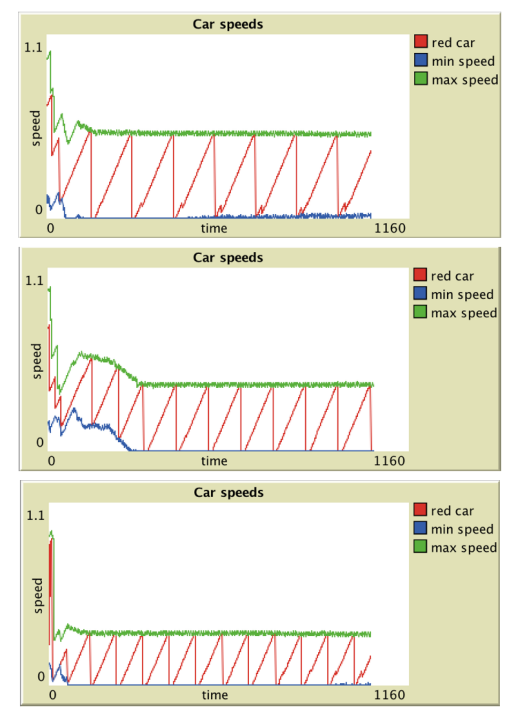

Fig. 4. The correlation between speed and space headway.

We conclude our discussion to emphasize how important a proper environment settings is. In fact, to determine the scale of the environment in which agents play is a fundamental stage in the development of any model. In the particular case of NetLogo, it is essential to define the maximum size of the environment in terms of a scale to perform all calculations involving distance. For instance, to model a road space of 1000 meters, an option would be to configure the space with 1000 patches which denotes a simple 1:1 scale. However, since a large number of patches affects the performance of the model, a configuration of a 1:5 scale would be more advisable. In this way, the road space would be represented with 200 patches. 


\section{Conclusions and Future Work}

We focus our attention in the basic elements to be considered in vehicular traffic by using modeling based on agents. By making use of a basic traffic model, we present the empirical relationships of a vehicular traffic system in terms of density, flow and speed. We also show how relevant the microscopic and macroscopic characteristics of vehicular traffic flows are for developing a realistic traffic model. Furthermore, the shortcomings of a basic traffic model were discussed to emphasize how important an appropriated representation of distance and time is for a traffic simulation system.

Many dynamic aspects of traffic flows have a huge influence over the developing a realistic traffic model. Dynamic and complex aspects to be modeled such as the conditions of the road (e.g. wet roads) and human factors (e.g. reaction time) are undoubtedly interesting aspects to investigate and to include in the developing of a traffic simulation system.

\section{References}

1. Bell, M., Bonsall, P., Leake, G., May, A., Nash, C.: Transport Planning and Traffic Engineering. Butterwörth-Heinemann, Elsevier (2006)

2. Greengard, S.: Smart Transportation Networks Drive Gains. Communications of the ACM 58(1), 25-27 (2015)

3. Holland, J.: Hidden Order: How Adaptation Builds Complexity. Helix Books/Addison-Wesley (1995)

4. Janota, A., Rastocny, K.: Multi-agent Approach to Traffic Simulation in NetLogo Environment Level Crossing Model. In: 5th International Conference Transport Systems Telematics TST05- 2005 (2005)

5. Kauffman, S.: At Home in the Universe: The Search for the Laws of SelfOrganization and Complexity. Oxford University Press (1995)

6. Lighthill, M., Whitham, G.: On kinematic waves: II. A theory of traffic flow on long crowded roads. In: Proceedings of the Royal Society. vol. A229, pp. 317-345 (1955)

7. Maerivoet, S., De Moor, B.: Traffic Flow Theory. eprint arXiv:physics/0507126 (2005)

8. Mayrhofer, C.: Performance, Scale and Time in Agent-based Traffic Modelling with NetLogo. Journal for Geographic Information Scien 1, 567-570 (2015)

9. Nagel, K., Schreckenberg, M.: A cellular automaton model for freeway traffic. Journal de Physique I France 2, 2221-2229 (1992)

10. Prigogine, I., Herman, R.: Kinetic Theory of Vehicular Traffic. Elsevier, New York (1971)

11. Richards, P.I.: Shockwaves on the highway. Operations Research 4, 42-51 (1956)

12. Wardrop, J.: Some theoretical aspects of road traffic research. In: Proceedings of the Institution of Civil Engineers. vol. 1 (1952)

13. Wilensky, U.: NetLogo Traffic Basic Model. Center for Connected Learning and Computer-Based Modeling, Northwestern University, Evanston, IL (1997), http://ccl.northwestern.edu/netlogo/models/TrafficBasic

14. Wilensky, U.: NetLogo. Center for Connected Learning and ComputerBased Modeling, Northwestern University, Evanston, IL (1999), http://ccl.northwestern.edu/netlogo/ 


\section{Diego Uribe, Luis Lugo, Enrique Cuan}

15. Wilensky, U., Rand, W.: An Introduction to Agent-Based Modeling. The MIT Press. Cambridge Massachusetts (2015) 